OPEN ACCESS

Edited by:

Maria Dolores Ganfornina,

University of Valladolid, Spain

Reviewed by:

Roland Strong,

Fred Hutchinson Cancer Research

Center, United States

Fernanda Marques,

University of Minho, Portugal

${ }^{*}$ Correspondence:

Kyoungho Suk

ksuk@knu.ac.kr

Specialty section:

This article was submitted to Integrative Physiology, a section of the journal

Frontiers in Physiology

Received: 05 December 2020

Accepted: 19 January 2021

Published: 05 February 2021

Citation:

Bhusal A, Lee W-H and Suk K (2021) Lipocalin-2 in Diabetic Complications of the Nervous System: Physiology, Pathology, and Beyond.

Front. Physiol. 12:638112.

doi: 10.3389/fphys.2021.638112

\section{Lipocalin-2 in Diabetic Complications of the Nervous System: Physiology, Pathology, and Beyond}

\author{
Anup Bhusal ${ }^{1,2}$, Won-Ha Lee ${ }^{3}$ and Kyoungho Suk ${ }^{1,2,4 *}$ \\ ${ }^{1}$ Department of Pharmacology, School of Medicine, Kyungpook National University, Daegu, South Korea, ${ }^{2} B K 21$ Plus KNU \\ Biomedical Convergence Program, Department of Biomedical Science, School of Medicine, Kyungpook National University, \\ Daegu, South Korea, ${ }^{3}$ School of Life Sciences, BK21 FOUR KNU Creative BioResearch Group, Kyungpook National \\ University, Daegu, South Korea, ${ }^{4}$ Brain Science and Engineering Institute, Kyungpook National University, Daegu, \\ South Korea
}

Lipocalin-2 (LCN2) is a $25 \mathrm{kDa}$ secreted protein that belongs to the family of lipocalins, a group of transporters of small hydrophobic molecules such as iron, fatty acids, steroids, and lipopolysaccharide in circulation. LCN2 was previously found to be involved in iron delivery, pointing toward a potential role for LCN2 in immunity. This idea was further validated when LCN2 was found to limit bacterial growth during infections in mice by sequestering iron-laden siderophores. Recently, LCN2 was also identified as a critical regulator of energy metabolism, glucose and lipid homeostasis, and insulin function. Furthermore, studies using Lcn2 knockout mice suggest an important role for LCN2 in several biobehavioral responses, including cognition, emotion, anxiety, and feeding behavior. Owing to its expression and influence on multiple metabolic and neurological functions, there has emerged a great deal of interest in the study of relationships between LCN2 and neurometabolic complications. Thorough investigation has demonstrated that LCN2 is involved in several neurodegenerative diseases, while more recent studies have shown that LCN2 is also instrumental for the progression of diabetic complications like encephalopathy and peripheral neuropathy. Preliminary findings have shown that LCN2 is also a promising drug target and diagnostic marker for the treatment of neuropathic complications from diabetes. In particular, future translational research related to LCN2, such as the development of small-molecule inhibitors or neutralizing antibodies against LCN2, appears essential for exploring its potential as a therapeutic target.

Keywords: lipocalin-2, immunity, energy metabolism, diabetes, neuroinflammation, diabetic complications, nervous system

\section{INTRODUCTION}

Diabetes is a syndrome defined by the presence of abnormally high blood glucose levels or hyperglycemia (Guthrie and Guthrie, 2004; Skyler et al., 2017). Hyperglycemia is known to cause a wide variety of metabolic disturbances and affect both the peripheral nervous system (PNS) and central nervous system (CNS), either directly or indirectly, which can lead to 
several complications collectively referred to as diabetic neuropathy over a long period of time (Sima et al., 2004; Malone, 2016). Diabetes mainly affects nerves in hands, feet, legs, and arms and is long considered a disease of the PNS. However, there is now increasing evidence of diabetic effects on the CNS as part of a condition called diabetic encephalopathy (Selvarajah et al., 2011). The pathogenesis of diabetic encephalopathy has not been fully defined, yet appears to show similarities to the progression of diabetic peripheral neuropathy (DPN) (Manschot et al., 2008).

Recently, there has been wide agreement that excessive glial activation is a key mechanism in both CNS- and PNS-related complications of diabetes involving the release of proinflammatory cytokines. Glial cells play an essential role in maintaining the normal function of nervous tissues. In the PNS, neurons are intimately associated with numerous glial cells; the neuronal soma is enclosed by satellite glial cells (SGCs) and axons are covered along their length by Schwann cells (Goncalves et al., 2018). Similarly, neurons are in close contact with astrocytes, microglia, oligodendrocytes, and Müller glial cells in the CNS. These glial cells are responsible for the uptake and metabolism of glucose in the nervous system. Fluctuations in glucose levels activate them, causing activation of MAPK/PI3K/Akt/NF- $\mathrm{BB}$ signaling and release of proinflammatory factors (QuincozesSantos et al., 2017; Hsieh et al., 2019). This inflammatory activation of glial cells triggers further metabolic deterioration and affects both small and large nerve fibers, resulting in nerve dysfunction that is characteristic of diabetic neuropathy (Goldberg, 2009; Pop-Busui et al., 2016).

Lipocalin-2 (LCN2), also known as neutrophil gelatinaseassociated lipocalin (NGAL), siderocalin, and $24 \mathrm{p} 3$, is a member of the lipocalin superfamily and is a pleiotropic mediator of various physiological and pathological processes (Xiao et al., 2017; Bhusal et al., 2019b). LCN2 so far is known to act through two major membrane-bound receptors: megalin, also known as low-density lipoprotein-related protein 2 (LRP2), and 24p3R, also referred to as solute carrier SLC22A17 or brain-type organic cation transporter (BOCT). LCN2 functions through these receptors in an iron-dependent manner, where apo-LCN2 chelates iron inside the cell, releases to the extracellular medium, reduces intracellular iron concentration, and finally causes cellular apoptosis. On the other hand, holo-LCN2 increases intracellular iron concentration and prevents cellular apoptosis by decreasing the expression of the proapoptotic protein Bcl-2-like protein 11 (Bim) (Devireddy et al., 2005). However, this hypothesis has lately been challenged, as HeLa cells expressing BOCT receptors did not exhibit cellular iron efflux following LCN2 treatment. Moreover, LCN2, even at higher doses, did not induce apoptosis in hematopoietic cell line (Correnti et al., 2012). Studies also failed to show the interaction between LCN2 and BOCT (Bennett et al., 2011; Correnti et al., 2012). Similarly, conflicting views have also been reported regarding the role of LCN2 in several metabolic conditions, as reviewed earlier by our group (Bhusal et al., 2019b). In light of these findings, LCN2 has recently been proposed to play an important role in the development of diabetic complications. Here, we review the physiological as well as pathological role of LCN2 in the nervous system, and discuss the latest reports as to how it could be used as a target for the treatment of diabetic neurological complications. In this review, the term "diabetic neuropathy" is used throughout the manuscript in reference to a disease of the peripheral and central nervous system, unless otherwise specified.

\section{THE ROLE OF LCN2 IN NERVOUS SYSTEM PHYSIOLOGY AND PATHOLOGY}

Lipocalin-2 is produced by mammalian hosts to bind bacterial siderophores and sequester free iron as part of innate immune defenses against bacterial infection; however, thus far, its role in the nervous system is less well understood. The upregulation of LCN2 in the brain was first observed in response to peripheral turpentine-induced inflammation (Liu and Nilsen-Hamilton, 1995). In addition, the study of LCN2 function in various neuroinflammatory conditions largely began with the observation of LCN2 expression in microglia (Lee et al., 2007) and astrocytes (Lee et al., 2009) in the CNS. However, the physiological role of LCN2 has not been studied due to its low or undetectable expression in healthy adult brains (Ip et al., 2011; Chakraborty et al., 2012; Kim et al., 2017; Kang et al., 2018) or peripheral nerves (Jeon et al., 2013; Bhusal et al., 2020), although some studies have demonstrated LCN2 protein expression in the hippocampus (Mucha et al., 2011; Chia et al., 2015; Furukawa et al., 2017), cortex, and amygdala (Furukawa et al., 2017) of normal rodents. Furthermore, strong constitutive expression of the LCN2 receptor $24 \mathrm{p} 3 \mathrm{R}$ in the brain of normal mice has been reported (Ip et al., 2011; Chia et al., 2015). It is, therefore, possible that basal expression of LCN2 in different regions of the brain may help in the defense of the CNS against pathogens. Beyond resisting infections, high levels of LCN2 in other regions of the brain may contribute to iron transport in these regions under normal conditions.

\section{LCN2 in Stress, Anxiety, Depression, and Cognitive Function}

Recent studies using Lcn 2 knockout (KO) animals have improved understanding of the role of LCN2 in the regulation of physiological conditions like stress, emotion, and memory. In one such study, Lcn2 $\mathrm{KO}$ mice displayed increased anxiety and depressive-like behaviors and mild spatial reference memory impairments (Ferreira et al., 2013). These altered phenotypes were associated with hyperactivation of the hypothalamicpituitary-adrenal axis, reflected in the increased levels of corticosteroids at both the morning and night periods in the Lcn2-deficient mice. Furthermore, the hippocampal neuronal morphology of Lcn2 KO mice displayed hypertrophy of granular and pyramidal neurons at the ventral hippocampus, a region implicated in emotional behavior, as well as neuronal atrophy at the dorsal hippocampus, a region implicated in memory and cognition (Ferreira et al., 2013). Another study by the 
same group found that $L c n 2$ deficiencies lead to higher proportion of progenitor cells in hippocampus exiting the cell cycle and progressing toward apoptotic cell death (Ferreira et al., 2018). Furthermore, deletion of $L c n 2$ in neural stem cells induced endogenous oxidative stress, cell cycle arrest, and cell death in an iron-mediated manner (Ferreira et al., 2018). In a later extension of their study, the impaired hippocampal neurogenesis observed in Lcn2 $\mathrm{KO}$ mice was relieved by voluntary running, which counteracted oxidative stress and promoted cell cycling of neural stem cells, resulting in the partial reduction of anxiety and improved contextual behavior (Ferreira et al., 2019). In line with this, the ablation of Lcn2 gene proved deleterious and promoted a stress-induced increase in spine density, which correlated with higher excitability of CA1 neurons and stressinduced anxiety (Mucha et al., 2011).

\section{LCN2 in Food Intake Regulation}

Mosialou et al. (2017) recently revealed an unexpected role of LCN2 regarding feeding behavior of mice. In that study, LCN2 secreted from bone crosses the blood-brain barrier, binds to melanocortin 4 receptors (MC4R) in neurons of the hypothalamus, and activates an MC4R-dependent anorexigenic pathway (Mosialou et al., 2017). This finding was extended to baboon, macaque, and human, where LCN2 acted as a satiety factor, and failure to stimulate postprandial LCN2 in individuals with obesity contributed to metabolic dysregulation (Petropoulou et al., 2020).

However, these observations should be carefully considered. In the study by Guo et al. (2010), indirect calorimetry measurements revealed no difference in food intake behavior between wild-type and Lcn2 KO mice. In another study, Lcn2deficient mice displayed no alteration in food intake upon Celastrol treatment, which is known to increase LCN2 levels in hypothalamus (Feng et al., 2019). Recently, LCN2overexpressing transgenic mice showed an increased food intake (Principi et al., 2019). Similarly, food intake was increased when mice were injected with LCN2 protein (Paton et al., 2013). These controversial findings warrant further investigation into the mechanisms how LCN2 regulates food intake behavior.

\section{LCN2 in Inflammatory and Other Neurological Disorders}

Beyond the physiological roles of LCN2, recent studies have shown an increase in expression of and an important role for LCN2 in various pathological states. LCN2 has been found to regulate diverse cellular processes and phenotypes in the nervous system, including cell death and survival (Lee et al., 2007; Naude et al., 2012; Bi et al., 2013), cell migration and morphology (Lee et al., 2011, 2012; Rathore et al., 2011), and the functional polarization of microglia (Jang et al., 2013b; McPherson et al., 2014) and astrocytes (Jang et al., 2013a). These functional characteristics of LCN2 have been exploited by many researchers to study the role of LCN2 in different neurological disorders, including neuroinflammation (Lee et al., 2011; Jin et al., 2014a, 2018; Mondal et al., 2020), Alzheimer's disease (Naude et al., 2012; Dekens et al., 2018;
Eruysal et al., 2019), ischemic stroke (Jin et al., 2014b; Xing et al., 2014; Hochmeister et al., 2016; Ranjbar Taklimie et al., 2019; Zhao et al., 2019), experimental autoimmune encephalomyelitis (Berard et al., 2012; Marques et al., 2012; Nam et al., 2014), brain and spinal cord injuries (Chia et al., 2011; Rathore et al., 2011; Dong et al., 2013), malignant gliomas (Suk, 2012), and pain hypersensitivity (Poh et al., 2012; Jeon et al., 2013; Jha et al., 2013, 2014). As a result of its active participation in the pathogenesis of various neurological diseases, LCN2 can be considered a promising therapeutic target for both prognostic and diagnostic purposes. Recently, the number of studies describing a role for LCN2 in metabolic homeostasis (Yan et al., 2007; Jun et al., 2011; Guo et al., 2012, 2016; Mosialou et al., 2020) and the pathogenesis of diabetes-related complications is on the rise, indicating similar importance of LCN2 in diabetes-related neurological disorders.

\section{LCN2 IN NEUROLOGICAL COMPLICATIONS OF DIABETES}

Recently, several clinical studies have demonstrated a close relationship between LCN2 expression and the risk of impaired glucose metabolism (Wang et al., 2007; Yang et al., 2009; Huang et al., 2012). Furthermore, LCN2 levels have been linked to diabetic complications like retinopathy (Chung et al., 2016; Wang et al., 2020) and nephropathy (Wu et al., 2014; Papadopoulou-Marketou et al., 2017; Sisman et al., 2020). However, the precise mechanisms underlying the role of LCN2 in diabetic complications remain unclear and several plausible explanations have been suggested. LCN2 has been reported to deliver iron to cells, causing intracellular iron overload and results in oxidative stress, cellular degeneration, and increased levels of advanced glycation end-product (AGE) receptors for AGE binding (Ciudin et al., 2010). LCN2 has been shown to activate metalloproteinase-9 (MMP-9) by forming a stable complex with MMP-9 (Yan et al., 2001). MMP-9 activation may then facilitate an increase in vascular permeability through the proteolytic degradation of tight junction proteins (Opdenakker and Abu El-Asrar, 2019). LCN2 may also be linked to the production of AGE (Chung et al., 2013; Petrica et al., 2015), which interacts with plasma membrane-localized receptors for AGEs (RAGE) to alter intracellular signaling, gene expression, and the release of proinflammatory molecules and free radicals (Singh et al., 2014). In addition, LCN2 is involved in immune reactions and inflammatory processes (Lee et al., 2011; Jin et al., 2014a, 2018; Mondal et al., 2020). These mechanisms involving LCN2 are all relevant in the nervous system, as LCN2 is significantly expressed in both CNS and PNS following the onset of diabetes, making LCN2 a potential focus in the study of diabetic neuropathy.

\section{LCN2 in Diabetic Encephalopathy}

Diabetic encephalopathy is a chronic complication of diabetes mellitus characterized by oxidative stress, impaired 
microvascular permeability, neurogenesis, cognitive functions, and electrophysiological, neurochemical, and structural abnormalities (Cai et al., 2011; Ho et al., 2013; Chen et al., 2018). People with diabetes mellitus have increased levels of proinflammatory cytokines such as C-reactive protein, TNF- $\alpha$, and IL-6 (Esposito et al., 2002; Tangvarasittichai et al., 2016). Recently, a study reported the upregulation and pathological role of LCN2 in the hippocampus of an insulin-deficient diabetes model created by streptozotocin injection (Bhusal et al., 2019a). In this study, deletion of the Lcn2 gene ameliorated diabetes-induced reactive gliosis and expression of proinflammatory cytokines in the hippocampus of diabetic mice. Moreover, Lcn2 KO diabetic mice showed decreased neuronal loss in the hippocampus compared to wild-type diabetic animals, an effect correlated with improved cognitive behavior (Bhusal et al., 2019a) (Figure 1). Previously, the same group reported increased plasma levels in patients with mild cognitive impairments (Choi et al., 2011), supporting the correlation between LCN2 and cognitive deficits. Another study conducted using four independent cohorts with a large number of samples concluded that cerebrospinal fluid LCN2 is a promising biochemical marker for the differential diagnosis of neurodegenerative dementias (Llorens et al., 2020). These findings indicate that LCN2 has significant translational potential for several brain-related complications of diabetes.

Recently, a study reported increased serum and hippocampal LCN2 levels in ob/ob mice. In this study, ob/ob mice showed impaired spatial learning behavior, which was improved following a calorie-restricted diet that correlated with decreased expression of LCN2 in the hippocampus (Park et al., 2019). Another study suggested that increasing LCN2-mediated iron uptake may be required for Toll-like receptor 4/endosome-related WD repeat and FYVE domain-containing 1 (Wdfyl)-signaling during hippocampal neuroinflammation in ob/ob mice (Jin et al., 2020). Furthermore, a study using a combination of high-fat diet and fructose showed that elevated hippocampal and peripheral LCN2 levels mediate the impact of chronic inflammation on the CNS, which is associated with behavioral dysfunction (de Sousa Rodrigues et al., 2017). Additionally, recent studies attempted to evaluate the role of a chronic high-fat diet in neuroinflammation with respect to myeloid sirtuin1 (SIRT1) and LCN2 function. It was found that SIRT1 promotes hippocampal inflammation in association with LCN2 levels (Kim et al., 2018; Park et al., 2019).

Obesity and high-fat diet are associated with an increased risk of developing insulin resistance and type 2 diabetes. Considering evidence that circulating LCN2 levels are associated with hyperglycemia, insulin resistance, and metabolic syndrome based on in vitro (Yan et al., 2007; Chan et al., 2016), in vivo (Wang et al., 2007; Guo et al., 2010), and clinical studies (Cakal et al., 2011), it is speculated that LCN2 may be involved in the regulation of insulin sensitivity in the brain, although the relationship between LCN2 expression and insulin resistance in the brain has not been investigated. Taken together, specific mechanisms

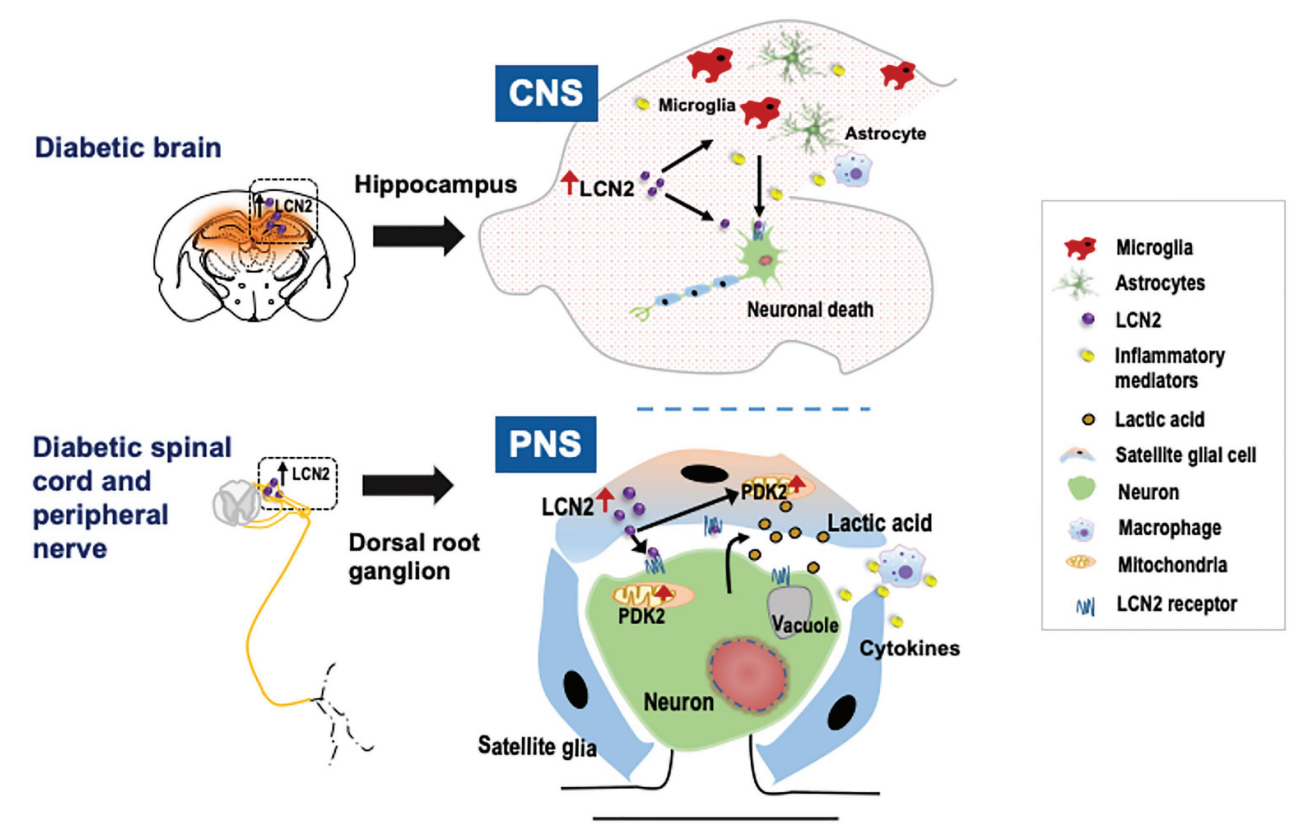

FIGURE 1 | Schematic representation of the role of lipocalin-2 (LCN2) in diabetic neuropathy. Diabetes leads to the upregulation of LCN2 in the hippocampus of the brain and in satellite glial cells (SGCs) of the dorsal root ganglion (DRG). In the central nervous system (CNS), induction of LCN2 causes activation of glial cells, macrophage infiltration, and upregulation of inflammatory cytokines leading to diabetic encephalopathy. In the peripheral nervous system (PNS), it has been suggested that LCN2 released from the SGCs in the DRG acts on SGCs as well as nearby neurons to induce pyruvate dehydrogenase kinase-2 (PDK2) expression. The LCN2-PDK2 axis-mediated glycolytic metabolic shift in the DRG results in the production of lactic acid, which causes an acidic microenvironment that eventually causes neuronal damage, leading to diabetic peripheral neuropathy. 
relating to the association between LCN2 and IR in the brain can be additional factors aggravating diabetic encephalopathy.

\section{LCN2 in Diabetic Peripheral Neuropathy}

Diabetic peripheral neuropathy is the most common microvascular complication of diabetes mellitus characterized by inflammation, oxidative stress, and mitochondrial dysfunction (Roman-Pintos et al., 2016). Based on the previous observations of the induction of LCN2 expression and its role in neuroinflammation in the brains of mice with diabetes or various demyelinating diseases (Nam et al., 2014; Chun et al., 2015; Al Nimer et al., 2016), Bhusal et al. (2020) have recently provided evidence for a potentially novel role of LCN2 in the progression of DPN. They found that LCN2 is expressed by SGCs in the dorsal root ganglion (DRG) and by Schwann cells in the sciatic nerves of diabetic mice. This LCN2 expression was dependent on high glucose levels, as evidenced by the decrease in LCN2 levels in the DRG and improvement in nerve conduction velocity in diabetic mice following insulin treatment. Furthermore, their study using Lcn $2 \mathrm{KO}$ mice showed a decrease in inflammation in the DRG and sciatic nerve and reduction in the consequent DPN phenotype; however, the mechanisms through which Lcn2 deficiency attenuates DPN remain unclear.

Pyruvate dehydrogenase kinase-2 (PDK2), a mitochondrial enzyme, is known to drive a metabolic shift in dorsal root ganglia, which induces neuroinflammation, lactic acid build-up and ultimately produces painful DPN (Rahman et al., 2016). Similarly, multiple studies have reported that LCN2 increases mitochondrial reactive oxygen species, alters mitochondrial oxidative phosphorylation, and impairs overall mitochondrial activity (Yang et al., 2012; Song et al., 2018; Chella Krishnan et al., 2019). Given the proven role of LCN2 and PDK2 in mitochondrial activity, Bhusal et al. (2020) investigated whether there is any functional relationship between LCN2 and PDK2 in terms of neuroinflammation and lactic acid production in DPN. In their study, PDK2 overexpression using adenoviruses in the DRG in Lcn2 KO mice potentiated inflammation and DPN, which was not observed when LCN2 was overexpressed in $P d k 2$ KO mice. Furthermore, LCN2 increased expression of PDK2 in SGC culture, which was shown using pharmacological

\section{REFERENCES}

Al Nimer, F., Elliott, C., Bergman, J., Khademi, M., Dring, A. M., Aeinehband, S., et al. (2016). Lipocalin-2 is increased in progressive multiple sclerosis and inhibits remyelination. Neurol. Neuroimmunol. Neuroinflamm. 3:e191. doi: 10.1212/NXI.0000000000000191

Bennett, K. M., Liu, J., Hoelting, C., and Stoll, J. (2011). Expression and analysis of two novel rat organic cation transporter homologs, SLC22A17 and SLC22A23. Mol. Cell. Biochem. 352, 143-154. doi: 10.1007/s11010-011-0748-y

Berard, J. L., Zarruk, J. G., Arbour, N., Prat, A., Yong, V. W., Jacques, F. H., et al. (2012). Lipocalin 2 is a novel immune mediator of experimental autoimmune encephalomyelitis pathogenesis and is modulated in multiple sclerosis. Glia 60, 1145-1159. doi: 10.1002/glia.22342

Bhusal, A., Rahman, M. H., Lee, W. H., Bae, Y. C., Lee, I. K., and Suk, K. (2019b). Paradoxical role of lipocalin-2 in metabolic disorders and neurological complications. Biochem. Pharmacol. 169:113626. doi: 10.1016/j.bcp.2019.113626 blockade to be dependent on peroxisome proliferator-activated receptors. From these findings, the authors concluded that LCN2 acts as an upstream regulator of PDK2 in the SGCs of DRG, which potentiates neuroinflammation, lactate surge, and consequent DPN (Bhusal et al., 2020) (Figure 1). These findings ultimately put forward a novel mechanism for an LCN2-PDK2lactic acid axis in diabetes-induced neuroinflammation and consequent diabetic complications like neuropathy.

\section{CONCLUDING REMARKS}

During the past few decades, there has been an increased understanding of the relationships among metabolic syndrome, adipokines, and inflammatory diseases. In this regard, LCN2 can be considered one of the mediators responsible for inflammation in complications associated with diabetes. Recent studies have provided essential evidence of LCN2 as a major player linked to metabolism, inflammation, and neuropathy. LCN2 expression may be a useful early diagnostic biomarker for diabetic neuropathy; however, further validation using human samples derived from larger multi-institutional cohorts is needed. Further research into the function of LCN2 will guide our understanding of its potential use as a diagnostic and therapeutic agent and will create new opportunities for improving the care of patients with diabetic neuropathy.

\section{AUTHOR CONTRIBUTIONS}

$\mathrm{AB}, \mathrm{W}-\mathrm{HL}$, and $\mathrm{KS}$ wrote the manuscript. All authors contributed to the article and approved the submitted version.

\section{FUNDING}

This work was supported by a grant from the Basic Science Research Program through the National Research Foundation (NRF), which is funded by the Korean government (MSIP; 2016M3C7A1904148, NRF-2017R1A5A2015391, and NRF-2020M3E5D9079764).
Bhusal, A., Rahman, M. H., Lee, W. H., Lee, I. K., and Suk, K. (2020). Satellite glia as a critical component of diabetic neuropathy: role of lipocalin-2 and pyruvate dehydrogenase kinase-2 axis in the dorsal root ganglion. Glia. doi: 10.1002/glia.23942 [Epub ahead of print]

Bhusal, A., Rahman, M. H., Lee, I. K., and Suk, K. (2019a). Role of hippocampal lipocalin-2 in experimental diabetic encephalopathy. Front. Endocrinol. 10:25. doi: 10.3389/fendo.2019.00025

Bi, F., Huang, C., Tong, J., Qiu, G., Huang, B., Wu, Q., et al. (2013). Reactive astrocytes secrete lcn2 to promote neuron death. Proc. Natl. Acad. Sci. U. S. A. 110, 4069-4074. doi: 10.1073/pnas.1218497110

Cai, X. J., Xu, H. Q., and Lu, Y. (2011). C-peptide and diabetic encephalopathy. Chin. Med. Sci. J. 26, 119-125. doi: 10.1016/ S1001-9294(11)60031-X

Cakal, E., Ozkaya, M., Engin-Ustun, Y., and Ustun, Y. (2011). Serum lipocalin-2 as an insulin resistance marker in patients with polycystic ovary syndrome. J. Endocrinol. Investig. 34, 97-100. doi: 10.1007/BF03347037 
Chakraborty, S., Kaur, S., Guha, S., and Batra, S. K. (2012). The multifaceted roles of neutrophil gelatinase associated lipocalin (NGAL) in inflammation and cancer. Biochim. Biophys. Acta 1826, 129-169. doi: 10.1016/j. bbcan.2012.03.008

Chan, Y. K., Sung, H. K., Jahng, J. W., Kim, G. H., Han, M., and Sweeney, G. (2016). Lipocalin-2 inhibits autophagy and induces insulin resistance in H9c2 cells. Mol. Cell. Endocrinol. 430, 68-76. doi: 10.1016/j.mce.2016.04.006

Chella Krishnan, K., Sabir, S., Shum, M., Meng, Y., Acin-Perez, R., Lang, J. M., et al. (2019). Sex-specific metabolic functions of adipose Lipocalin-2. Mol. Metab. 30, 30-47. doi: 10.1016/j.molmet.2019.09.009

Chen, R., Shi, J., Yin, Q., Li, X., Sheng, Y., Han, J., et al. (2018). Morphological and pathological characteristics of brain in diabetic encephalopathy. $J$. Alzheimers Dis. 65, 15-28. doi: 10.3233/JAD-180314

Chia, W. J., Dawe, G. S., and Ong, W. Y. (2011). Expression and localization of the iron-siderophore binding protein lipocalin 2 in the normal rat brain and after kainate-induced excitotoxicity. Neurochem. Int. 59, 591-599. doi: 10.1016/j.neuint.2011.04.007

Chia, W. J., Tan, F. C., Ong, W. Y., and Dawe, G. S. (2015). Expression and localisation of brain-type organic cation transporter (BOCT/24p3R/LCN2R) in the normal rat hippocampus and after kainate-induced excitotoxicity. Neurochem. Int. 87, 43-59. doi: 10.1016/j.neuint.2015.04.009

Choi, J., Lee, H. W., and Suk, K. (2011). Increased plasma levels of lipocalin 2 in mild cognitive impairment. J. Neurol. Sci. 305, 28-33. doi: 10.1016/j. jns.2011.03.023

Chun, B. Y., Kim, J. H., Nam, Y., Huh, M. I., Han, S., and Suk, K. (2015). Pathological involvement of astrocyte-derived lipocalin-2 in the demyelinating optic neuritis. Invest. Ophthalmol. Vis. Sci. 56, 3691-3698. doi: 10.1167/iovs.15-16851

Chung, T. W., Choi, H. J., Kim, C. H., Jeong, H. S., and Ha, K. T. (2013). Lipocalin-2 elicited by advanced glycation end-products promotes the migration of vascular smooth muscle cells. Biochim. Biophys. Acta 1833, 3386-3395. doi: 10.1016/j.bbamcr.2013.10.011

Chung, J. O., Park, S. Y., Cho, D. H., Chung, D. J., and Chung, M. Y. (2016). Plasma neutrophil gelatinase-associated lipocalin levels are positively associated with diabetic retinopathy in patients with type 2 diabetes. Diabet. Med. 33, 1649-1654. doi: 10.1111/dme.13141

Ciudin, A., Hernandez, C., and Simo, R. (2010). Iron overload in diabetic retinopathy: a cause or a consequence of impaired mechanisms? Exp. Diabetes Res. 2010:714108. doi: 10.1155/2010/714108

Correnti, C., Richardson, V., Sia, A. K., Bandaranayake, A. D., Ruiz, M., Suryo Rahmanto, Y., et al. (2012). Siderocalin/Lcn2/NGAL/24p3 does not drive apoptosis through gentisic acid mediated iron withdrawal in hematopoietic cell lines. PLoS One 7:e43696. doi: 10.1371/journal.pone.0043696

de Sousa Rodrigues, M. E., Bekhbat, M., Houser, M. C., Chang, J., Walker, D. I., Jones, D. P., et al. (2017). Chronic psychological stress and high-fat highfructose diet disrupt metabolic and inflammatory gene networks in the brain, liver, and gut and promote behavioral deficits in mice. Brain Behav. Immun. 59, 158-172. doi: 10.1016/j.bbi.2016.08.021

Dekens, D. W., Naude, P. J. W., Keijser, J. N., Boerema, A. S., De Deyn, P. P., and Eisel, U. L. M. (2018). Lipocalin 2 contributes to brain iron dysregulation but does not affect cognition, plaque load, and glial activation in the J20 Alzheimer mouse model. J. Neuroinflammation 15:330. doi: 10.1186/ s12974-018-1372-5

Devireddy, L. R., Gazin, C., Zhu, X., and Green, M. R. (2005). A cell-surface receptor for lipocalin $24 \mathrm{p} 3$ selectively mediates apoptosis and iron uptake. Cell 123, 1293-1305. doi: 10.1016/j.cell.2005.10.027

Dong, M., Xi, G., Keep, R. F., and Hua, Y. (2013). Role of iron in brain lipocalin 2 upregulation after intracerebral hemorrhage in rats. Brain Res. 1505, 86-92. doi: 10.1016/j.brainres.2013.02.008

Eruysal, E., Ravdin, L., Kamel, H., Iadecola, C., and Ishii, M. (2019). Plasma lipocalin-2 levels in the preclinical stage of Alzheimer's disease. Alzheimers Dement. 11, 646-653. doi: 10.1016/j.dadm.2019.07.004

Esposito, K., Nappo, F., Marfella, R., Giugliano, G., Giugliano, F., Ciotola, M., et al. (2002). Inflammatory cytokine concentrations are acutely increased by hyperglycemia in humans: role of oxidative stress. Circulation 106, 2067-2072. doi: 10.1161/01.CIR.0000034509.14906.AE

Feng, X., Guan, D., Auen, T., Choi, J. W., Salazar-Hernandez, M. A., Faruk, F., et al. (2019). Lipocalin 2 does not play a role in celastrol-mediated reduction in food intake and body weight. Sci. Rep. 9:12809. doi: 10.1038/ s41598-019-49151-8
Ferreira, A. C., Novais, A., Sousa, N., Sousa, J. C., and Marques, F. (2019). Voluntary running rescues the defective hippocampal neurogenesis and behaviour observed in lipocalin 2-null mice. Sci. Rep. 9:1649. doi: 10.1038/ s41598-018-38140-y

Ferreira, A. C., Pinto, V., Da Mesquita, S., Novais, A., Sousa, J. C., Correia-Neves, M., et al. (2013). Lipocalin-2 is involved in emotional behaviors and cognitive function. Front. Cell. Neurosci. 7:122. doi: 10.3389/fncel.2013.00122

Ferreira, A. C., Santos, T., Sampaio-Marques, B., Novais, A., Mesquita, S. D., Ludovico, P., et al. (2018). Lipocalin-2 regulates adult neurogenesis and contextual discriminative behaviours. Mol. Psychiatry 23, 1031-1039. doi: 10.1038/mp.2017.95

Furukawa, T., Shimoyama, S., Miki, Y., Nikaido, Y., Koga, K., Nakamura, K., et al. (2017). Chronic diazepam administration increases the expression of Lcn 2 in the CNS. Pharmacol. Res. Perspect. 5:e00283. doi: 10.1002/prp2.283

Goldberg, R. B. (2009). Cytokine and cytokine-like inflammation markers, endothelial dysfunction, and imbalanced coagulation in development of diabetes and its complications. J. Clin. Endocrinol. Metab. 94, 3171-3182. doi: 10.1210/jc.2008-2534

Goncalves, N. P., Vaegter, C. B., and Pallesen, L. T. (2018). Peripheral glial cells in the development of diabetic neuropathy. Front. Neurol. 9:268. doi: 10.3389/fneur.2018.00268

Guo, H., Foncea, R., O’Byrne, S. M., Jiang, H., Zhang, Y., Deis, J. A., et al. (2016). Lipocalin 2, a regulator of retinoid homeostasis and retinoid-mediated thermogenic activation in adipose tissue. J. Biol. Chem. 291, 11216-11229. doi: $10.1074 /$ jbc.M115.711556

Guo, H., Jin, D., Zhang, Y., Wright, W., Bazuine, M., Brockman, D. A., et al. (2010). Lipocalin-2 deficiency impairs thermogenesis and potentiates dietinduced insulin resistance in mice. Diabetes 59, 1376-1385. doi: 10.2337/ db09-1735

Guo, H., Zhang, Y., Brockman, D. A., Hahn, W., Bernlohr, D. A., and Chen, X. (2012). Lipocalin 2 deficiency alters estradiol production and estrogen receptor signaling in female mice. Endocrinology 153, 1183-1193. doi: 10.1210/ en.2011-1642

Guthrie, R. A., and Guthrie, D. W. (2004). Pathophysiology of diabetes mellitus. Crit. Care Nurs. Q. 27, 113-125. doi: 10.1097/00002727-200404000-00003

Ho, N., Sommers, M. S., and Lucki, I. (2013). Effects of diabetes on hippocampal neurogenesis: links to cognition and depression. Neurosci. Biobehav. Rev. 37, 1346-1362. doi: 10.1016/j.neubiorev.2013.03.010

Hochmeister, S., Engel, O., Adzemovic, M. Z., Pekar, T., Kendlbacher, P., Zeitelhofer, M., et al. (2016). Lipocalin-2 as an infection-related biomarker to predict clinical outcome in ischemic stroke. PLoS One 11:e0154797. doi: 10.1371/journal.pone.0154797

Hsieh, C. F., Liu, C. K., Lee, C. T., Yu, L. E., and Wang, J. Y. (2019). Acute glucose fluctuation impacts microglial activity, leading to inflammatory activation or self-degradation. Sci. Rep. 9:840. doi: 10.1038/s41598-018-37215-0

Huang, Y., Yang, Z., Ye, Z., Li, Q., Wen, J., Tao, X., et al. (2012). Lipocalin-2, glucose metabolism and chronic low-grade systemic inflammation in Chinese people. Cardiovasc. Diabetol. 11:11. doi: 10.1186/1475-2840-11-11

Ip, J. P., Nocon, A. L., Hofer, M. J., Lim, S. L., Muller, M., and Campbell, I. L. (2011). Lipocalin 2 in the central nervous system host response to systemic lipopolysaccharide administration. J. Neuroinflammation 8:124. doi: 10.1186/ 1742-2094-8-124

Jang, E., Kim, J. H., Lee, S., Kim, J. H., Seo, J. W., Jin, M., et al. (2013a). Phenotypic polarization of activated astrocytes: the critical role of lipocalin- 2 in the classical inflammatory activation of astrocytes. J. Immunol. 191, 5204-5219. doi: $10.4049 /$ jimmunol.1301637

Jang, E., Lee, S., Kim, J. H., Kim, J. H., Seo, J. W., Lee, W. H., et al. (2013b). Secreted protein lipocalin-2 promotes microglial M1 polarization. FASEB J. 27, 1176-1190. doi: 10.1096/fi.12-222257

Jeon, S., Jha, M. K., Ock, J., Seo, J., Jin, M., Cho, H., et al. (2013). Role of lipocalin-2-chemokine axis in the development of neuropathic pain following peripheral nerve injury. J. Biol. Chem. 288, 24116-24127. doi: 10.1074/jbc. M113.454140

Jha, M. K., Jeon, S., Jin, M., Lee, W. H., and Suk, K. (2013). Acute phase protein lipocalin-2 is associated with formalin-induced nociception and pathological pain. Immune Netw. 13, 289-294. doi: 10.4110/in.2013.13.6.289

Jha, M. K., Jeon, S., Jin, M., Ock, J., Kim, J. H., Lee, W. H., et al. (2014). The pivotal role played by lipocalin-2 in chronic inflammatory pain. Exp. Neurol. 254, 41-53. doi: 10.1016/j.expneurol.2014.01.009 
Jin, M., Jang, E., and Suk, K. (2014a). Lipocalin-2 acts as a neuroinflammatogen in lipopolysaccharide-injected mice. Exp. Neurobiol. 23, 155-162. doi: 10.5607/ en.2014.23.2.155

Jin, Z., Jung, Y., Yi, C. O., Lee, J. Y., Jeong, E. A., Lee, J. E., et al. (2018). Atorvastatin pretreatment attenuates kainic acid-induced hippocampal neuronal death via regulation of lipocalin-2-associated neuroinflammation. Korean J. Physiol. Pharmacol. 22, 301-309. doi: 10.4196/kjpp.2018.22.3.301

Jin, M., Kim, J. H., Jang, E., Lee, Y. M., Soo Han, H., Woo, D. K., et al. (2014b). Lipocalin-2 deficiency attenuates neuroinflammation and brain injury after transient middle cerebral artery occlusion in mice. J. Cereb. Blood Flow Metab. 34, 1306-1314. doi: 10.1038/jcbfm.2014.83

Jin, Z., Kim, K. E., Shin, H. J., Jeong, E. A., Park, K. A., Lee, J. Y., et al. (2020). Hippocampal lipocalin 2 is associated with neuroinflammation and iron-related oxidative stress in ob/ob mice. J. Neuropathol. Exp. Neurol. 79, 530-541. doi: 10.1093/jnen/nlaa017

Jun, L. S., Siddall, C. P., and Rosen, E. D. (2011). A minor role for lipocalin 2 in high-fat diet-induced glucose intolerance. Am. J. Physiol. Endocrinol. Metab. 301, E825-E835. doi: 10.1152/ajpendo.00147.2011

Kang, S. S., Ren, Y., Liu, C. C., Kurti, A., Baker, K. E., Bu, G., et al. (2018). Lipocalin-2 protects the brain during inflammatory conditions. Mol. Psychiatry 23, 344-350. doi: 10.1038/mp.2016.243

Kim, K. E., Jeong, E. A., Lee, J. Y., Yi, C. O., Park, K. A., Jin, Z., et al. (2018). Myeloid sirtuin1 deficiency aggravates hippocampal inflammation in mice fed high-fat diets. Biochem. Biophys. Res. Commun. 499, 1025-1031. doi: 10.1016/j.bbrc.2018.04.044

Kim, J. H., Ko, P. W., Lee, H. W., Jeong, J. Y., Lee, M. G., Kim, J. H., et al. (2017). Astrocyte-derived lipocalin-2 mediates hippocampal damage and cognitive deficits in experimental models of vascular dementia. Glia 65, 1471-1490. doi: 10.1002/glia.23174

Lee, S., Kim, J. H., Kim, J. H., Seo, J. W., Han, H. S., Lee, W. H., et al. (2011). Lipocalin-2 is a chemokine inducer in the central nervous system: role of chemokine ligand 10 (CXCL10) in lipocalin-2-induced cell migration. J. Biol. Chem. 286, 43855-43870. doi: 10.1074/jbc.M111.299248

Lee, S., Lee, J., Kim, S., Park, J. Y., Lee, W. H., Mori, K., et al. (2007). A dual role of lipocalin 2 in the apoptosis and deramification of activated microglia. J. Immunol. 179, 3231-3241. doi: 10.4049/jimmunol.179.5.3231

Lee, S., Lee, W. H., Lee, M. S., Mori, K., and Suk, K. (2012). Regulation by lipocalin-2 of neuronal cell death, migration, and morphology. J. Neurosci. Res. 90, 540-550. doi: 10.1002/jnr.22779

Lee, S., Park, J. Y., Lee, W. H., Kim, H., Park, H. C., Mori, K., et al. (2009). Lipocalin-2 is an autocrine mediator of reactive astrocytosis. J. Neurosci. 29, 234-249. doi: 10.1523/JNEUROSCI.5273-08.2009

Liu, Q., and Nilsen-Hamilton, M. (1995). Identification of a new acute phase protein. J. Biol. Chem. 270, 22565-22570. doi: 10.1074/jbc.270.38.22565

Llorens, F., Hermann, P., Villar-Pique, A., Diaz-Lucena, D., Nagga, K., Hansson, O., et al. (2020). Cerebrospinal fluid lipocalin 2 as a novel biomarker for the differential diagnosis of vascular dementia. Nat. Commun. 11:619. doi: 10.1038/ s41467-020-14373-2

Malone, J. I. (2016). Diabetic central neuropathy: CNS damage related to hyperglycemia. Diabetes 65, 355-357. doi: 10.2337/dbi15-0034

Manschot, S. M., Biessels, G. J., Rutten, G. E., Kessels, R. P., Gispen, W. H., Kappelle, L. J., et al. (2008). Peripheral and central neurologic complications in type 2 diabetes mellitus: no association in individual patients. J. Neurol. Sci. 264, 157-162. doi: 10.1016/j.jns.2007.08.011

Marques, F., Mesquita, S. D., Sousa, J. C., Coppola, G., Gao, F., Geschwind, D. H., et al. (2012). Lipocalin 2 is present in the EAE brain and is modulated by natalizumab. Front. Cell. Neurosci. 6:33. doi: 10.3389/ fncel.2012.00033

McPherson, C. A., Merrick, B. A., and Harry, G. J. (2014). In vivo molecular markers for pro-inflammatory cytokine M1 stage and resident microglia in trimethyltin-induced hippocampal injury. Neurotox. Res. 25, 45-56. doi: 10.1007/s12640-013-9422-3

Mondal, A., Bose, D., Saha, P., Sarkar, S., Seth, R., Kimono, D., et al. (2020). Lipocalin 2 induces neuroinflammation and blood-brain barrier dysfunction through liver-brain axis in murine model of nonalcoholic steatohepatitis. $J$. Neuroinflammation 17:201. doi: 10.1186/s12974-020-01876-4

Mosialou, I., Shikhel, S., Liu, J. M., Maurizi, A., Luo, N., He, Z., et al. (2017). MC4R-dependent suppression of appetite by bone-derived lipocalin 2. Nature 543, 385-390. doi: 10.1038/nature21697
Mosialou, I., Shikhel, S., Luo, N., Petropoulou, P. I., Panitsas, K., Bisikirska, B., et al. (2020). Lipocalin-2 counteracts metabolic dysregulation in obesity and diabetes. J. Exp. Med. 217:e20191261. doi: 10.1084/jem.20191261

Mucha, M., Skrzypiec, A. E., Schiavon, E., Attwood, B. K., Kucerova, E., and Pawlak, R. (2011). Lipocalin-2 controls neuronal excitability and anxiety by regulating dendritic spine formation and maturation. Proc. Natl. Acad. Sci. U. S. A. 108, 18436-18441. doi: 10.1073/pnas.1107936108

Nam, Y., Kim, J. H., Seo, M., Kim, J. H., Jin, M., Jeon, S., et al. (2014). Lipocalin-2 protein deficiency ameliorates experimental autoimmune encephalomyelitis: the pathogenic role of lipocalin-2 in the central nervous system and peripheral lymphoid tissues. J. Biol. Chem. 289, 16773-16789. doi: $10.1074 /$ jbc.M113.542282

Naude, P. J., Nyakas, C., Eiden, L. E., Ait-Ali, D., van der Heide, R., Engelborghs, S., et al. (2012). Lipocalin 2: novel component of proinflammatory signaling in Alzheimer's disease. FASEB J. 26, 2811-2823. doi: 10.1096/f.11-202457

Opdenakker, G., and Abu El-Asrar, A. (2019). Metalloproteinases mediate diabetes-induced retinal neuropathy and vasculopathy. Cell. Mol. Life Sci. 76, 3157-3166. doi: 10.1007/s00018-019-03177-3

Papadopoulou-Marketou, N., Margeli, A., Papassotiriou, I., Chrousos, G. P., Kanaka-Gantenbein, C., and Wahlberg, J. (2017). NGAL as an early predictive marker of diabetic nephropathy in children and young adults with type 1 diabetes mellitus. J. Diabetes Res. 2017:7526919. doi: 10.1155/2017/7526919

Park, K. A., Jin, Z., An, H. S., Lee, J. Y., Jeong, E. A., Choi, E. B., et al. (2019). Effects of caloric restriction on the expression of lipocalin-2 and its receptor in the brown adipose tissue of high-fat diet-fed mice. Korean J. Physiol. Pharmacol. 23, 335-344. doi: 10.4196/kjpp.2019.23.5.335

Paton, C. M., Rogowski, M. P., Kozimor, A. L., Stevenson, J. L., Chang, H., and Cooper, J. A. (2013). Lipocalin-2 increases fat oxidation in vitro and is correlated with energy expenditure in normal weight but not obese women. Obesity 21, E640-E648. doi: 10.1002/oby.20507

Petrica, L., Vlad, A., Gluhovschi, G., Zamfir, A., Popescu, C., Gadalean, F., et al. (2015). Glycated peptides are associated with proximal tubule dysfunction in type 2 diabetes mellitus. Int. J. Clin. Exp. Med. 8, 2516-2525.

Petropoulou, P. I., Mosialou, I., Shikhel, S., Hao, L., Panitsas, K., Bisikirska, B., et al. (2020). Lipocalin-2 is an anorexigenic signal in primates. eLife 9:e58949. doi: 10.7554/eLife.58949

Poh, K. W., Yeo, J. F., Stohler, C. S., and Ong, W. Y. (2012). Comprehensive gene expression profiling in the prefrontal cortex links immune activation and neutrophil infiltration to antinociception. J. Neurosci. 32, 35-45. doi: 10.1523/JNEUROSCI.2389-11.2012

Pop-Busui, R., Ang, L., Holmes, C., Gallagher, K., and Feldman, E. L. (2016). Inflammation as a therapeutic target for diabetic neuropathies. Curr. Diab. Rep. 16:29. doi: 10.1007/s11892-016-0727-5

Principi, E., Buschiazzo, A., Papait, A., Castagnola, P., Costa, D., Pece, R., et al. (2019). Anthropometric and glucometabolic changes in an aged mouse model of lipocalin-2 overexpression. Int. J. Obes. 43, 189-201. doi: 10.1038/ s41366-018-0171-5

Quincozes-Santos, A., Bobermin, L. D., de Assis, A. M., Goncalves, C. A., and Souza, D. O. (2017). Fluctuations in glucose levels induce glial toxicity with glutamatergic, oxidative and inflammatory implications. Biochim. Biophys. Acta Mol. basis Dis. 1863, 1-14. doi:10.1016/j.bbadis.2016.09.013

Rahman, M. H., Jha, M. K., Kim, J. H., Nam, Y., Lee, M. G., Go, Y., et al. (2016). Pyruvate dehydrogenase kinase-mediated glycolytic metabolic shift in the dorsal root ganglion drives painful diabetic neuropathy. J. Biol. Chem. 291, 6011-6025. doi: 10.1074/jbc.M115.699215

Ranjbar Taklimie, F., Gasterich, N., Scheld, M., Weiskirchen, R., Beyer, C., Clarner, T., et al. (2019). Hypoxia induces astrocyte-derived lipocalin-2 in ischemic stroke. Int. J. Mol. Sci. 20:1271. doi: 10.3390/ijms20061271

Rathore, K. I., Berard, J. L., Redensek, A., Chierzi, S., Lopez-Vales, R., Santos, M., et al. (2011). Lipocalin 2 plays an immunomodulatory role and has detrimental effects after spinal cord injury. J. Neurosci. 31, 13412-13419. doi: 10.1523/ JNEUROSCI.0116-11.2011

Roman-Pintos, L. M., Villegas-Rivera, G., Rodriguez-Carrizalez, A. D., Miranda-Diaz, A. G., and Cardona-Munoz, E. G. (2016). Diabetic polyneuropathy in type 2 diabetes mellitus: inflammation, oxidative stress, and mitochondrial function. J. Diabetes Res. 2016:3425617. doi: 10.1155/2016/3425617

Selvarajah, D., Wilkinson, I. D., Davies, J., Gandhi, R., and Tesfaye, S. (2011). Central nervous system involvement in diabetic neuropathy. Curr. Diab. Rep. 11, 310-322. doi: 10.1007/s11892-011-0205-z 
Sima, A. A., Kamiya, H., and Li, Z. G. (2004). Insulin, C-peptide, hyperglycemia, and central nervous system complications in diabetes. Eur. J. Pharmacol. 490, 187-197. doi: 10.1016/j.ejphar.2004.02.056

Singh, V. P., Bali, A., Singh, N., and Jaggi, A. S. (2014). Advanced glycation end products and diabetic complications. Korean J. Physiol. Pharmacol. 18, 1-14. doi: 10.4196/kjpp.2014.18.1.1

Sisman, P., Gul, O. O., Dirican, M., Bal, A. S., Cander, S., and Erturk, E. (2020). Urinary neutrophil gelatinase-associated lipocalin (NGAL) as a marker of diabetic nephropathy in type 1 diabetic patients. Clin. Lab. 66. doi: 10.7754/Clin.Lab.2019.190326

Skyler, J. S., Bakris, G. L., Bonifacio, E., Darsow, T., Eckel, R. H., Groop, L., et al. (2017). Differentiation of diabetes by pathophysiology, natural history, and prognosis. Diabetes 66, 241-255. doi: 10.2337/db16-0806

Song, E., Ramos, S. V., Huang, X., Liu, Y., Botta, A., Sung, H. K., et al. (2018). Holo-lipocalin-2-derived siderophores increase mitochondrial ROS and impair oxidative phosphorylation in rat cardiomyocytes. Proc. Natl. Acad. Sci. U. S. A. 115, 1576-1581. doi: 10.1073/pnas.1720570115

Suk, K. (2012). Proteomic analysis of glioma chemoresistance. Curr. Neuropharmacol. 10, 72-79. doi: 10.2174/157015912799362733

Tangvarasittichai, S., Pongthaisong, S., and Tangvarasittichai, O. (2016). Tumor necrosis factor-alpha, interleukin-6, C-reactive protein levels and insulin resistance associated with type 2 diabetes in abdominal obesity women. Indian J. Clin. Biochem. 31, 68-74. doi: 10.1007/s12291-015-0514-0

Wang, Y., Lam, K. S., Kraegen, E. W., Sweeney, G., Zhang, J., Tso, A. W., et al. (2007). Lipocalin-2 is an inflammatory marker closely associated with obesity, insulin resistance, and hyperglycemia in humans. Clin. Chem. 53, 34-41. doi: 10.1373/clinchem.2006.075614

Wang, H., Lou, H., Li, Y., Ji, F., Chen, W., Lu, Q., et al. (2020). Elevated vitreous Lipocalin-2 levels of patients with proliferative diabetic retinopathy. BMC Ophthalmol. 20:260. doi: 10.1186/s12886-020-01462-5

Wu, C., Wang, Q., Lv, C., Qin, N., Lei, S., Yuan, Q., et al. (2014). The changes of serum sKlotho and NGAL levels and their correlation in type 2 diabetes mellitus patients with different stages of urinary albumin. Diabetes Res. Clin. Pract. 106, 343-350. doi: 10.1016/j.diabres.2014.08.026

Xiao, X., Yeoh, B. S., and Vijay-Kumar, M. (2017). Lipocalin 2: an emerging player in iron homeostasis and inflammation. Annu. Rev. Nutr. 37, 103-130. doi: 10.1146/annurev-nutr-071816-064559
Xing, C., Wang, X., Cheng, C., Montaner, J., Mandeville, E., Leung, W., et al. (2014). Neuronal production of lipocalin-2 as a help-me signal for glial activation. Stroke 45, 2085-2092. doi: 10.1161/ STROKEAHA.114.005733

Yan, L., Borregaard, N., Kjeldsen, L., and Moses, M. A. (2001). The high molecular weight urinary matrix metalloproteinase (MMP) activity is a complex of gelatinase B/MMP-9 and neutrophil gelatinase-associated lipocalin (NGAL). Modulation of MMP-9 activity by NGAL. J. Biol. Chem. 276, 37258-37265. doi: 10.1074/jbc.M106089200

Yan, Q. W., Yang, Q., Mody, N., Graham, T. E., Hsu, C. H., Xu, Z., et al. (2007). The adipokine lipocalin 2 is regulated by obesity and promotes insulin resistance. Diabetes 56, 2533-2540. doi: 10.2337/db07-0007

Yang, B., Fan, P., Xu, A., Lam, K. S., Berger, T., Mak, T. W., et al. (2012). Improved functional recovery to $\mathrm{I} / \mathrm{R}$ injury in hearts from lipocalin-2 deficiency mice: restoration of mitochondrial function and phospholipids remodeling. Am. J. Transl. Res. 4, 60-71.

Yang, Y. H., He, X. J., Chen, S. R., Wang, L., Li, E. M., and Xu, L. Y. (2009). Changes of serum and urine neutrophil gelatinase-associated lipocalin in type-2 diabetic patients with nephropathy: one year observational follow-up study. Endocrine 36, 45-51. doi: 10.1007/s12020-009-9187-x

Zhao, N., Xu, X., Jiang, Y., Gao, J., Wang, F., Xu, X., et al. (2019). Lipocalin-2 may produce damaging effect after cerebral ischemia by inducing astrocytes classical activation. J. Neuroinflammation 16:168. doi: 10.1186/ s12974-019-1556-7

Conflict of Interest: The authors declare that the research was conducted in the absence of any commercial or financial relationships that could be construed as a potential conflict of interest.

Copyright (c) 2021 Bhusal, Lee and Suk. This is an open-access article distributed under the terms of the Creative Commons Attribution License (CC BY). The use, distribution or reproduction in other forums is permitted, provided the original author(s) and the copyright owner(s) are credited and that the original publication in this journal is cited, in accordance with accepted academic practice. No use, distribution or reproduction is permitted which does not comply with these terms. 